\title{
THE SPECTRUM OF ORTHOGONAL SUMS OF SUBNORMAL PAIRS
}

\author{
by K. RUDOL
}

(Received 20 August 1985; revised 29 October, 1986)

Introduction. This note provides yet another example of the difficulties that arise when one wants to extend the spectral theory of subnormal operators to subnormal tuples. Several basic properties of a subnormal operator $Y$ remain true for tuples; e.g. the existence and uniqueness of its minimal normal extension $N$, the spectral inclusion $\sigma(N) \subset \sigma(Y)$-proved for $n$-tuples in [4] and generalized to infinite tuples in [5]. However, neither the invariant subspace theorem nor the spectral mapping theorem in the "strong form" as in [3] is known so far for subnormal tuples.

The present note shows that even such a well known equality as

$$
\sigma\left(\bigoplus_{n=1}^{\infty} Y_{n}\right)=\left(\bigcup_{n=1}^{\infty} \sigma\left(Y_{n}\right)\right)^{-}
$$

valid for bounded sequences $\left\{Y_{n}\right\}$ of subnormals fails to have a multiparameter analogue. Namely, we shall construct a sequence of subnormal pairs $\left(S_{n}, T_{n}\right)$ for which the equality (1) below fails.

$$
\sigma\left(\oplus S_{n}, \oplus T_{n}\right)=\left(\bigcup \sigma\left(S_{n}, T_{n}\right)\right)^{-} .
$$

Here $\sigma$ stands for the joint spectrum in the sense of J. L. Taylor [8, 2], the bar denotes closure in the natural topology of $\mathbb{C}^{2}$. By subnormal we mean a pair being a restriction of two commuting, bounded normal operators to one of their common invariant subspaces.

Remarks. The containment " $\supset$ " in (1) does always take place. However, each of the following conditions suffices for the equality there for pairs, or even tuples of arbitrary commuting operators.

(a) The sequence $\left(S_{n}, T_{n}\right)$ is constant beginning from some $k$ : for all $n \geqslant k$.

(b) $\left\|S_{n}\right\| \rightarrow 0$ and $\left\|T_{n}\right\| \rightarrow 0$ as $n \rightarrow \infty$.

(c) $S_{n}=T_{n}$ and the $S_{n}$ are subnormal for $n$ large enough.

(To prove (b) use the semicontinuity of $\sigma$, cf. [6]. (c) follows from $\left({ }^{*}\right)$ if we identify $\sigma(S, S)$ with $\sigma(S)$.)

It would be interesting to know more nontrivial sufficient conditions for the equality in (1), since this equality may be applied to solving certain linear equations, a technique developed in the proof of our main result.

The author expresses his gratitude to Professors Jan Janas and Jan Stochel for helpful conversations and for finding numerous mistakes in the former version of this note.

Preliminaries. Let $S$ and $T$ be a pair of bounded, commuting operators on a Hilbert Glasgow Math. J. 30 (1988) 11-15. 
space $H$. Then, by definition, $(0.0) \notin \sigma(S, T)$ iff the mappings: $f \rightarrow S f \oplus T f$ and $(g \oplus h) \rightarrow S g-T h$ form a short exact sequence $0 \rightarrow H \rightarrow H \oplus H \rightarrow H \rightarrow 0$. If this is the case, then the "Laplacian" $X:=S S^{*}+T T^{*}$ is invertible and for $A:=S^{*} X^{-1}, B:=T^{*} X^{-1}$ we have

$$
S A+T B=I,
$$

where $I$ denotes the identity operator on $H$. See [2]. Conversely, if (2) has a solution with $A, B$ in a commutative algebra containing $S$ and $T$ then $(0,0) \notin \sigma(S, T)$. Generally, $\sigma(S, T)$ is defined as $\left\{(z, w) \in \mathbb{C}^{2} ;(0,0) \in \sigma(S-z I, T-w I)\right\}$.

If $S, T$ are multiplication operators on a Hardy space, the equality (2) looks like a solution of the corona equation. This formal similarity lies behind a deep relationship between (1) and the corona problem; cf. [5]. In that setting instead of Taylor's joint spectrum I have considered the so called extended spectrum of subnormal representations of $H^{\infty}(\Omega)$, the algebra of all bounded analytic functions on a (Runge) domain $\Omega$ in $\mathbb{C}^{2}$. Then (1) for that type of spectrum is equivalent to the corona theorem for $H^{\infty}(\Omega)$ and so is not always true. This follows from N. Sibony's counterexample, given in [7]. Here we use the following modification of this example: there is a Runge domain $G$ contained in the unit bidisc $\mathbb{D}^{2}$ such that $\mathbb{D}^{2} \notin G$ but $H^{\infty}(G)=H^{\infty}\left(\mathbb{D}^{2}\right)$ i.e., any $f \in H^{\infty}(G)$ extends analytically onto $\mathbb{D}^{2}$ with the same norm. The construction in [7] is simple.

Choose a discrete subset $\alpha=\left\{\alpha_{m} ; m<\infty\right\}$ of the unit disc $\mathbb{D}$ such that $|u(z)| \leqslant$ $\sup _{m}\left|u\left(\alpha_{m}\right)\right|$ for all $u \in H^{\infty}(\mathbb{D}), z \in \mathbb{D}$. Next construct a non-negative bounded subharmonic function $V$ on $\mathbb{D}$ such that $\alpha=V^{-1}\{0\}$. Let $G:=\left\{(z, w) \in \mathbb{D}^{2} ;|w|<\exp (-V(z))\right\}$; then

$$
H^{\infty}(G)=H^{\infty}\left(\mathbb{D}^{2}\right)
$$

However, we need here such an extension for some Hilbert space of analytic functions on $G$ in place of $H^{\infty}(G)$. Unfortunately, I cannot prove that this extension takes place for the Hardy or Bergman space over $G$, but only for the Lumer-Hardy space $L H^{2}(G)$ which does not seem to be a Hilbert space. To overcome this difficulty I introduce some technical $L^{2}$-norms.

The construction. Let $G$ be as in (3) a domain related to the set $\alpha=\cup_{n=2}^{\infty} C_{n}$, where each $C_{n}$ is a collection of $n^{4}$ points equidistributed at the circle $|z|=1-(1 / n)$. Choose an exhaustion of $G$ by a sequence of smoothly bordered domains of holomorphy $Q_{n}$ (cf. [5]) such that $\bar{Q}_{n} \subset Q_{n+1} \subset \ldots \subset G=\cup Q_{n}$ and for which $(z, w) \in Q_{n}$ if either $z \in C_{n},|w| \leqslant 1-(1 / n)$ or if $|z| \leqslant 1-(1 / 2 n)$ and $|w| \leqslant \frac{1}{4}$.

\section{Notation:}

$v \quad$ volume i.e. 4-dimensional Lebesgue measure on $\mathbb{C}^{2}$

$\mu_{n}$ the equidistributed probability measure on $C_{n}$ and

$v^{\prime}$ the planar Lebesgue measure on $\mathbb{C}$. 
Let us define the measure $v_{n}$ on $Q_{n}$ by the following formula:

$$
\int f d v_{n}=\int_{Q_{n}} f d v+\int_{C_{n}} d \mu_{n}(z) \int_{|w|<1-(1 / n)} f(z, w) d v^{\prime}(w) .
$$

As $\left(S_{n}, T_{n}\right)$, we shall take the multiplication by $z$ and by $w$ operators on the space $H_{n}$, defined as the closure in $L^{2}\left(v_{n}\right)$ of all complex polynomials $p(z, w)$. In other words, $\left(S_{n} f\right)(z, w)=z f(z, w)$ and $\left(T_{n} f\right)(z, w)=w f(z, w)$. Note that $H_{n}$ is nothing else but the renormed Bergman space with a norm equivalent to the $L^{2}(v)$-norm. The point of this modification is that the norms of elements $h \in H_{n}$ will be more influenced as $n \rightarrow \infty$ by the values $h(z, w)$ for $z \in C_{n}$, helping to "enlarge" radii of convergence in the variable $w$. More precisely, for $0 \leqslant r \leqslant 1$, having a function $a$ analytic on $r \mathbb{D}$, put

$$
\|a\|_{r}:=\left(\int_{|w|<r}|a(w)|^{2} d v^{\prime}(w)\right)^{1 / 2} .
$$

Then for $s:=1-(1 / n), r:=1-(1 / 2 n)$ our basic estimate will be

$$
\|a\|_{s} \leqslant \pi\left(\int_{C_{n}}|a|^{2} d \mu_{n}\right)^{1 / 2}+C n^{-2}\|a\|_{r}
$$

where $C$ is independent of $n, a$. The proof of (5) will be given later on. Let us also note that if $a(w)=\sum a_{k} w^{k}$, then using polar coordinates one may easily estimate the Fourier coefficients $a_{k}$ as follows

$$
\left|a_{k}\right| \leqslant M_{k}\|a\|_{i},
$$

where $t=\frac{1}{4}$ and $M_{k}^{2}=4^{2 k+2}(k+1) / \pi$.

The key property of the sequence $\left\{H_{n}\right\}$ is contained in the following result.

LEMMA. If $f_{n} \in H_{n}$ form a sequence converging pointwise on $G$ with the $L^{2}\left(v_{n}\right)$-norms of $f_{n}$ bounded by some $M<\infty$, then $f:=\lim f_{n}$ extends analytically onto $\mathbb{D}^{2}$.

Assuming the lemma and (5) for a moment, we shall prove our main result.

PROPOSITION. The $\left(S_{n}, T_{n}\right)$ are subnormal pairs of contractions for which the equality (1) fails.

Proof. The subnormality is obvious-the same formulae define normal extensions on $L^{2}\left(v_{n}\right)$ of these pairs. $\left\|S_{n}\right\| \leqslant 1$, since $|z|<1$ on $Q_{n}$ and similarly $\left\|T_{n}\right\| \leqslant 1$. It is easy to see that $\sigma\left(S_{n}, T_{n}\right) \subset \bar{G}$. Indeed, for $\left(z^{\prime}, w^{\prime}\right) \notin \bar{G}$ there exist functions $f, g$ analytic on $G$ such that $\left(z-z^{\prime}\right) f+\left(w-w^{\prime}\right) g \equiv 1$. Therefore the equation (2) has solutions in the algebra of multiplication operators by functions from $H^{\infty}\left(Q_{n}\right)$ and the criterion $\left(2^{\prime}\right)$ is applicable (cf. [5]).

Let us fix a point $\left(z^{\prime}, w^{\prime}\right) \in \mathbb{D}^{2} \backslash \bar{G}$. If (1) were true, we would have $(0,0) \notin \sigma(S, T)$, where $S:=\oplus\left(S_{n}-z^{\prime} I\right), T:=\oplus\left(T_{n}-w^{\prime} I\right)$, and there would exist operators $X, A, B$ of the form $A=\oplus A_{n}, B=\oplus B_{n}$ with $\left\|A_{n}\right\|,\left\|B_{n}\right\|$ bounded, which would solve (2).

The constant polynomial 1 has its $L^{2}\left(v_{n}\right)$-norms bounded as $n \rightarrow \infty$ and so have the functions $f_{n}:=A_{n} 1$ and $g_{n}:=B_{n} 1$. We may apply a normal family argument and assume 
that these functions converge on $G$ to certain functions $f, g$ respectively. These functions satisfy $\left(z-z^{\prime}\right) f+\left(w-w^{\prime}\right) g=1$ (by (2)) for any $(z, w) \in G$ and, after analytic continuation (by the Lemma)-also for any $(z, w) \in \mathbb{D}^{2}$, which is absurd: taking $z=z^{\prime}, w=w^{\prime}$ we get $0=1$.

REMark. We do not know if the $A_{n}, B_{n}$ commute with $S_{n}$ and $T_{n}$. If this were true, it would be a major simplification as these operators would then be multiplications by $f_{n}$ and $g_{n}$, and this would provide the uniform estimates (on $Q_{n}$ ) for these functions. To conclude we need to prove (5) and the Lemma.

Proof. For $z \in \mathbb{C}$ with $|z|=s$ (i.e. $1-(1 / n)$ let $L_{z}$ be the point of $C_{n}$ next to $z$ in the clockwise direction of this circle. Then

$$
\left|z-L_{z}\right| \leqslant 2 \pi s n^{-4}:=K n^{-4} .
$$

If $r=1-(1 / 2 n)$ and $|w| \leqslant r$, the estimate on the derivative $a^{\prime}(w)$ treated as a Taylor coefficient gives $\left|a^{\prime}(w)\right| \leqslant K^{\prime} n^{2}\|a\|_{r}$. To see this, use (6) for the $L^{2}$-norm of $a$ over $\{z,|z-w|<r-|w|\}$ when $r-|w| \geqslant \frac{1}{2} n$. Hence

$$
\left|a(z)-a\left(L_{z}\right)\right| \leqslant K K^{\prime} n^{2-4}\|a\|_{r}:=K^{\prime \prime} n^{-2}\|a\|_{r} .
$$

Let $\mu$ be the normalized Lebesgue measure on the unit circle. Then it is easy to see that

$$
\int_{C_{n}}|a|^{2} d \mu_{n}=\int_{|z|=1}\left|a\left(L_{s z}\right)\right|^{2} d \mu .
$$

Denoting the square root of the last integral by $J$ and using (7) we get

$$
J \leqslant\left(\int_{C_{n}}|a|^{2} d \mu_{n}\right)^{1 / 2}+K^{\prime \prime} n^{-2}\|a\|_{r}
$$

Now the comparison between the Bergman and Hardy norms yields $\|a\|_{s} \leqslant \pi^{1 / 2} s J \leqslant \pi J$, which proves the estimate (5).

To prove the Lemma let us develop $f$ and $f_{n}$ in power series in the variable $w$, say $f(z, w)=\sum a_{k}(z) w^{k}$ for $(z, w) \in G$ and $f_{n}(z, w)=\sum a_{k, n}(z) w^{k}$ if $\{z\} \times(w \mathbb{D}) \subset Q_{n}$, (e.g. if $|z| \leqslant r,|w| \leqslant \frac{1}{4}$ or if $z \in C_{n}$ and $|w| \leqslant s$, where $r, s$ are as above).

Obviously, the functions $a_{k, n}$ are analytic on $|z|<1-(1 / 2 n)$ and converging to $a_{k}$ uniformly on compact subsets of $D$ since $f_{n} \rightarrow f$ along with all derivatives.

From (6) we obtain

$$
\left|a_{k, n}(z)\right|^{2} \leqslant M_{k}^{2} \int_{|w|<1 / 4}\left|f_{n}(z, w)\right|^{2} d v^{\prime}(w) .
$$

Integration over $r \mathbb{D}$ and the definition of $v_{n}$ gives the estimate $\left\|a_{k, n}\right\|_{r} \leqslant M_{k} M$, which is independent of $n$. Indeed, $r \mathbb{D} \times \frac{1}{4} \mathbb{D} \subset Q_{n}$ and $d v^{\prime}(z) d v^{\prime}(w)=d v(z, w)$. Similarly, the second term contributing to $v_{n}$ and (6) with $s$ in place of $t$ gives $\int_{C_{n}}\left|a_{k, n}\right|^{2} d \mu_{n} \leqslant$ $s^{-2 k}\left(M^{\prime} M\right)^{2}$. Now the application of (5) implies that $\left\|a_{k, n}\right\|_{s} \leqslant s^{-k} M^{\prime \prime}+C n^{-2} M_{k} M$. Note 
that $\|a\|_{t}$ is increasing and continuous with respect to $t$, so we may fix $s^{\prime}<1$ and, since $s=1-1 / n \rightarrow 1$, letting $n \rightarrow \infty$ we shall obtain $\left\|a_{k}\right\|_{s^{\prime}} \leqslant M^{\prime \prime}$. Because $s^{\prime}$ may be arbitrarily close to 1 , also $\left\|a_{k}\right\|_{1} \leqslant M^{\prime \prime}$. This, independent of $k$ estimate, guarantees the convergence of $\sum a_{k}(z) w^{k}$ for all $(z, w) \in \mathbb{D}^{2}$, proving the Lemma.

Note added in September 1986. The example obtained in this work can be used, as noted by J. Janas, to show the following: the description of the spectrum of inductive limits given in [9] for self-adjoint operators cannot be extended to inductive limits of subnormal pairs.

\section{REFERENCES}

1. J. B. Conway, Subnormal operators, (Pitman Publishing Co., 1981).

2. R. E. Curto, Fredholm and invertible $n$-tuples of operators. The deformation problem. Trans. Amer. Math. Soc. 266 (1981) 129-159.

3. J. Dudziak, Spectral mapping theorems for subnormal operators, J. Functional Analysis $\mathbf{5 6}$ (1984), 360-387.

4. M. Putinar, Spectral inclusion for subnormal $n$-tuples, Proc. Amer. Math. Soc. 90 (1984), 405-406.

5. K. Rudol, Extended spectrum of subnormal representations, Bull. Acad. Polon. Sci., Math. 31 (1983), 361-368.

6. K. Rudol, Spectral mapping theorems for analytic functional calculi, Operator Theory: Adv. Appl. 17 (1986), 331-340.

7. N. Sibony, Prolongement des fonctions holomorphes bornée et metrique de Carathéodory, Invent. Math. 29 (1975), 205-230.

8. J. L. Taylor, A joint spectrum of several commuting operators, J. Functional Analysis 6 (1970), 1-38.

9. A. V. Marchenko, Self-adjoint differential operators of an infinite number of variables, (in Russian) Mat. Sbornik (2) 96 (1975), 276-293.

InSTytut Matematyczny Pan

Kraków, u1. Solskiego 30

POLAND 